\title{
Qualitative Ansätze entwickeln
}

\section{Bei der Umsetzung Nachhaltiger Regionalentwicklung geht es zunehmend um die Nutzung von konkreten Projekterfahrungen in anderen Zusammenhängen. Es stellt sich die Frage nach den Wechselbeziehungen zwischen Durchführung und den Rahmenbedingungen eines Prozesses. Ein geeignetes Monitoring er- füllt aber auch gegenüber Öffentlichkeit und Auftraggebern wichtige Aufgaben.}

$\mathrm{B}$ Von Thomas Fiebig isher erfolgt die Strukturierung von Prozessen Nachhaltiger Regionalentwicklung maßgeblich durch die Entwicklung von Zielsystemen und ,--werten“. Sie werden aus dem Vergleich des Ist- und Sollzustandes der regionalen Entwicklung heraus aufgestellt und bestimmt (vgl. auch den Beitrag von Molitor in diesem Heft). Kerninstrumente sind dabei Programme, Gesetze, Pläne usw. Diese Elemente erweisen sich, zumal in dem hier in Rede stehenden thematischen Zusammenhang, als sehr statisch und im Verlaufe des Prozesses wenig anpassungsfähig. Außerdem können die Fortentwicklung und der Erfolg in der Regel nur qualitativ beschrieben werden. Eine Lösung für diese Nachteile in der Frage der Erfolgskon̂trolle wird oft in einem traditionellen Konzept der Planungsund Beobachtungsmethodik gesucht: der Darstellung von Entwicklungsprozessen anhand umfangreicher Indikatorensysteme mit zum Teil sehr unterschiedlichen Ausprägungen in Bezug auf Aussagetiefe und Erhebungsqualität (1).

Die Komplexität von nachhaltigen Entwicklungsprozessen führt bei diesen überwiegend quantifizierend ausgerichteten Modellen jedoch $\mathrm{zu}$ einem umständlich handhabbaren und oft unübersehbaren Katalog von Indikatoren, die gelegentlich mit dem Begriff Datenfriedhof belegt werden können. Für die unmittelbar in Prozesse nachhaltiger Regionalentwicklung einbezogenen Akteure und Zielgruppen sind solche Indikatorensysteme und Meßmethoden von geringerer Relevanz. Sie prallen dabei auf das Evaluierungsund Forschungsinteresse beteiligter und unbeteiligter Einrichtungen und Organisationen, indem Handeln und Verhalten an abstrakten Werten oder Zielgrößen bewertet und ausgerichtet werden sollen.

In der Diskussion über den verständlichen Wunsch nach Meßbarkeit von Wirkung und Erfolg Nachhaltiger Regionalentwicklung wird die qualitative Komponente der Darstellung der Prozesse oft übersehen. Eine ganze Reihe von Aspekten wie Motivation, Interessen und Kommunikation der Akteure lassen sich nicht oder nur indirekt abbilden. Wird der Prozeß Nachhaltiger Regionalentwicklung in seinem Zielsystem offen angelegt und erst durch die Beteiligten im Laufe des Kommunikations- und Konsultationsprozesses erstellt, dann ist die Forderung nach quantitativer Meßbarkeit noch weniger sinnvoll. Verschiedene sozial- und wirtschaftswissenschaftliche Ansätze bieten die Chance, diesen „Graubereich“ der Erfolgskontrolle weiterhin nutzbar zu halten. Teilnehmende Beobachtung, Evaluierung qualitativer Aspekte in Projekten und Programmen oder auch Konzepte des Controllings von Organisationsentwicklung und -kommunikation bieten vielfältige Möglichkeiten, mit denen Komponenten des Prozesses Nachhaltiger Regionalentwicklung überprüft werden können. Die Beobachtung bzw. das Monitoring muß dabei korrespondierend mit der Vorgehensweise im Prozeß entweder offen angelegt sein oder ein festumrissenes Gebäude von Fragen und Untersuchungsebenen haben. Es kann sich, und muß sich sogar zum Teil, aus verschiedenen sozial- und wirtschaftswissenschaftlichen Methodenansätzen bedienen: Interviews vermischen sich mit teilnehmender Beobachtung, verhaltensbezogene mit juristischen Fragen. Damit ist dieser Methoden-Mix ein Abbild der in Prozessen Nachhaltiger Regionalentwicklung einbezogenen Vielfalt von Akteuren und Gruppen. Dies wirkt nach außen unter Umständen wie eine methodische und inhaltliche Beliebigkeit. Das Monitoring hat jedoch damit den Vorteil, sich der Dynamik von Prozessen und Projekten anpassen zu können.

\section{Erfolgskontrolle sicherstellen}

Mit der Abkehr von quantitativen Meßmethoden und Indikatorensystemen ist keine Vernachlässi- gung des Aspektes der Erfolgskontrolle verbunden. Der Prozeß Nachhaltiger Regionalentwicklung kann grob in zwei Hauptphasen unterteilt werden. Die erste Phase des Prozeßbeginns ist geprägt durch viele Fragen, Ungeduld und $\mathrm{z}$. T. (hektischen) Aktionismus. In ihr wird versucht, den Elan des Anfangs zu konservieren. In der zweiten Phase werden Akteure und Gruppen ins Boot geholt, die Projekte und Initiativen umsetzen.

An dieser einfachen Aufteilung wird deutlich, wie wichtig Zwischenaufnahmen und ein Monitoring des Prozesses sind: Die ständige Überprüfung des Entwicklungsstandes des Prozesses und die sich daraus ableitenden „Korrekturen“ sind erste Ansätze einer Erfolgskontrolle. Sie sind Voraussetzung für die Übertragbarkeit von Konzepten Nachhaltiger Entwicklung auf andere Regionen und ein erster Schritt der Operationalisierung.

Dabei kann das Monitoring streng genommen nicht ausschließlich objektiv sein. Leistungskriterien und Erfolgsfaktoren von Prozessen Nachhaltiger Regionalentwicklung sind nur zum Teil objektiv bestimmt (Zielsysteme, Indikatorensysteme usw.). Sie sind zu einem nicht unerheblichen Teil personenbezogen und subjektiv, wenn es um die Mitwirkungs- und Kooperationsbereitschaft oder Interessenskongruenzen geht. Fehlt dem Konzept an dieser Stelle eine durchgängige Systematik in der Vorgehensweise und Umsetzung, so kann es auch nicht die eine Methode des Monitorings geben.

\section{Monitoring und Akzeptanz}

Monitoring kann aber auch in einem anderen Zusammenhang gesehen werden. Es dient der Sicherung von Ergebnissen und Wirkungen von Maßnahmen und leistet damit einen Beitrag zur „Nachhaltigkeit“. Monitoring kann Akzeptanzprobleme des Prozesses Nachhaltiger Regionalentwicklung vermindern. Die Skepsis gegenüber neuen, selbstbestimmteren Prozessen und die (v.a. zu Beginn) notwendige Überzeugungsarbeit lassen sich mit Hilfe des Monitorings auf die wichtigsten Kernfragen konzentrieren.

Im Mittelpunkt des Monitorings stehen neben der ,inhaltlichen Systembildung“ die „organisatorische Systembildung" zur Durchführung von Prozessen und Projekten Nachhaltiger Regionalentwicklung. Die zentralen Aufgaben in dieser "Systembildung" werden in der Regel von Kommunikation organisierenden und Entscheidungsprozessen koordinierenden Institutionen wahrgenommen(2). Die bisherigen 
Abbildung 1: Rolle und Aufgaben des Monitorings in Prozessen Nachhaltiger Regionalentwicklung

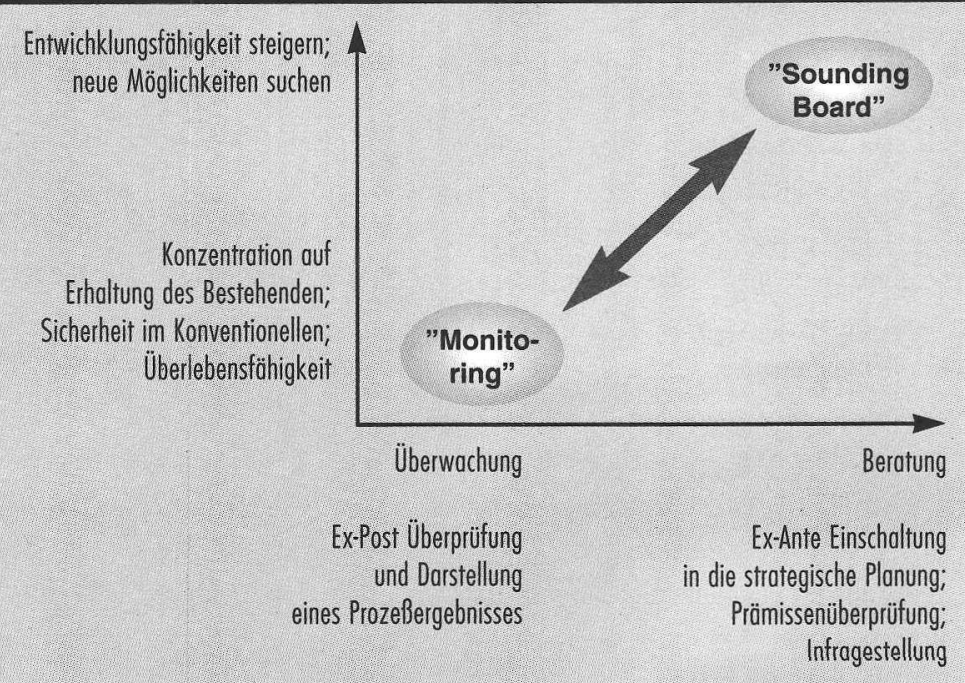

Quelle: Veränderte und vom Autor ergänzte Darstellung nach K. Bleicher: "Das Konzept Integriertes Management", Frankfurt a. M. / New York 1995, S. 147.

Umsetzungserfahrungen zeigen mittlerweile die weitere Entwicklung auf: Die Rolle der Selbstorganisation von Akteuren in einem ergebnisoffenen Prozeß wird zunehmen (3).

Damit wird die Erfolgsbestimmung und -trächtigkeit von Nachhaltiger Regionalentwicklung aber nicht einfacher. Im Gegenteil, es werden neue Fragen aufgeworfen: Was kann die Öffentlichkeit mit einem eingangs unstrukturierten und offenen Handlungskonzept anfangen? Wie können Akteure und Gruppen zum Erfolg von Projekten beitragen? Wo bleiben die fachbezogenen Verwaltungen und demokratischen Entscheidungsebenen? Drohen eine Aushöhlung und Unterminierung von Kompetenzen oder gar Legitimationskrisen? Langjährig bestehende Initiativen sehen ihre Projekte mit einem Mal von außen majorisiert usw..

\section{Fortentwicklung nötig}

Es múß also mit einer nicht unerheblichen Skepsis von verschiedenen Seiten gerechnet werden. Hier kann das Monitoring dazu dienen, Konflikte und Blockaden zu lösen und eine Selbststeuerung des Prozesses zu sichern. Damit kann Monitoring nicht mehr eine schematisierte, nachträgliche Überwachung der Wirkungen und des Erfolges bedeuten, wie es z. B. aus dem Methodenkontext der Betriebswirtschaftslehre her bekannt ist. Es muß sich stärker auf eine begleitende Beratung der Projektsteuerer und anderer Akteure einlassen und zu einem ,Sounding Board" fortentwickeln (vgl. Abb. 1).

Damit löst sich das Monitoring von im vorhinein bestimmten und statischen Zielsystemen und chendes", beratendes oder ,auslotendes "Monitoring begleiteten Prozesses können sich die Ziele ändern und anpassen. Diskussionen, ob nun eine Entwicklung ein Niveau erreicht hat, das „,besser“ oder ,schlechter“ im Sinne der Nachhaltigkeit $\mathrm{zu}$ bewerten ist, werden entschärft.

Die bisher praktizierte Berücksichtigung einer Vielzahl von Einzelzielen und Meßindikatoren (z. B. durch Abdeckung aller drei Nachhaltigkeitsebenen) wird der Komplexität der Abhängigkeiten und Beziehungen nicht gerecht. Das Monitoring sollte sich der Erfahrung anpassen, daß Prämissen und Ziele im Verlaufe des Prozesses in Frage gestellt werden müssen. Dies ist nur zum Teil einsehbar. Sind doch die intensiven Klärungs- und Kommunikationsprozesse $\mathrm{zu}$ Beginn einer Nachhaltigen Regionalentwicklung häufig auf ein festes Strategie-, Ziel- oder Indikatorensystem festgelegt und der Weg zur Einigung, zum Konsens sehr muihsam. Diese Systeme helfen zwar den Prozeß zu strukturieren und dienen der Umsetzung, lassen aber eine zentrale Frage unbeantwortet: Wie kann eine wirksame Verbindung zwischen den Strategien, den Zielen oder Meßzahlen zum individuellen Verhalten von Akteuren und Betroffenen hergestellt werden? Hierbei reicht es nicht, Prozesse und Projekte zu beschreiben und Prognosen aufzustellen. Dem Monitoring kommt in diesem Falle auch eine „didaktische Dimension“ zu, die es nicht allein durch statische Meß- und Prüfprogramme leisten kann. -katalogen. Während eines durch ein ,spre-

\section{Anmerkungen}

(1) Als Einführung, Überblick und Stand der Diskussion über Möglichkeiten von Indikatorensystemen geeignet sind z. B. M. Born: Indikatoren zur Nachhaltigen Entwicklung", econtur-Positionen 1 u. 2; Bremen 1997 und H. Diefenbacher et al.: Nachhaltige Wirtschaftsentwicklung im regionalen Bereich, Heidelberg 1997.

(2) Vgl. zur Rolle und Bedeutung von „intermediären Organisationen" U. Peters et al.: Nachhaltige Regionalentwicklung - ein neues Leitbild für eine veränderte Struktur- und Regionalpolitik, Trier 1996, S. 53 ff.

(3) W. Kleine-Limberg: Chancen und Grenzen regionaler Selbstorganisation. In: R. Danielzyk et al.: Von der eigenständigen zur Nachhaltigen Regionalentwicklung. econturPositionen Nr. 5, Bremen 1997, S. 25-33.

Der Autor
Thomas Fiebig ist freiberuflich in der Stadt-und
Regionalplanung und in der Entwicklung regionalö-
konomischer Konzepte tätig.
Kontakt: Hafenstrafe 13, 48153 Münster,
Tol. 0251 / 525369 , Fax 0251/ 525369 ,
E-Mail: t-fiebig@muenster.de

\section{Politische
obologie}

Endspurt

Die Ökologische Steuerreform vor dem Durchbruch?

Die Ökologische Steuerreform ist nicht zuletzt durch den umstrittenen Benzinpreis-Beschluß der Grünen wieder ein Thema öffentlichen Interesses. Das Ziel der Steuerreform, Energie zu verteuern und die Arbeit zu entlasten, stößt auf Zustimmung quer durch die Parteien, die Wirtschaft und die Wissenschaft. Der Weg dorthin ist allerdings umstritten.

Das aktuelle Heft der Politischen Ökologie bündelt die aktuellen Positionen, berichtet über Erfahrungen anderer Länder mit einer Ökologischen Steuerreform und beleuchtet die Konsequenzen für Staat, Wirtschaft und die Verbraucher. Und es zeigt, daß die Chancen, mit der Ökologischen Steuerreform einen wichtigen Hebel für ein zukunftsfähiges Deutschland zu etablieren, heute besser sind denn je.

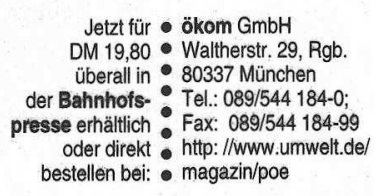


(c) 20I0 Authors; licensee IÖW and oekom verlag. This is an article distributed under the terms of the Creative Commons Attribution Non-Commercial No Derivates License (http://creativecommons.org/licenses/by-nc-nd/3.o/), which permits unrestricted use, distribution, and reproduction in any medium, provided the original work is properly cited. 